\title{
Artificial Neural Networks in Fruits: A Comprehensive Review
}

\author{
Sumit Goyal \\ Member, IDA, New Delhi, India \\ E-mail: thesumitgoyal@gmail.com
}

\begin{abstract}
This review discusses the application of artificial neural networks (ANN) modeling in fruits. It covers all fruits in which ANN modeling has been applied. ANN is quite a new and easy computational modeling approach used for prediction, which has become popular and accepted by food industry, researchers, scientists and students. ANNs have been applied in almost every field of science and technology, viz., speech synthesis \& recognition, pattern classification, adaptive interfaces between humans \& complex physical systems, clustering, function approximation, image data compression, non-linear system modeling, associative memory, combinatorial optimization, control and several others, as they have proved valuable tools for obtaining the required output. ANN provides an exciting alternative method for solving a variety of problems in different areas of science and engineering. The aim of this communication is to discover the recent advances of ANN technology implemented in fruits, and discuss the critical role that ANN plays in predictive modelling.
\end{abstract}

Index Terms - Artificial neural networks (ANN), machine learning, backpropagation, fruits, neurocomputing, soft computing

\section{INTRODUCTION}

The comprehensive use of artificial neural networks (ANN) in a variety of applications makes it an essential tool in the development of products that have implications for the human world. The role model for ANN is the human mind. ANN is a collection of methodologies that intend to utilize the endurance for ambiguity and uncertainty to achieve complete information and provide low cost solutions. ANN is playing an increasingly significant role in many application areas of science, engineering and technology.

ANN can be regarded as an extension of many classification techniques, which have been developed over several decades. These networks are inspired by the concept of the biological nervous system, and have proved to be robust in dealing with the ambiguous data and the kind of problems that require the interpolation of large amounts of data. Instead of sequentially performing a program of instructions, neural networks explore many hypotheses simultaneously using massive parallelism. Neural networks have the potential for solving problems in which some inputs and corresponding output values are known, but the relationship between the inputs and outputs is not well understood or is difficult to translate into a mathematical function. These conditions are commonly found in tasks involving grading, sorting and identifying agricultural products [1-6].

In present era, the consumers are extremely conscious about quality of the foods they buy. Regulatory agencies are also very vigilant about quality and safety issues and insist on the manufacturers adhering to the label claims about quality and shelf life. Such discerning consumers, therefore, pose a far greater challenge in product development and marketing. Till date no such review is present in the academic literature, which describes the use of ANN in fruits. This communication reports and discusses a wide variety of fruits, viz., apple, apple juice, avocado, apricot, banana, blackberry, blackcurrant, blueberries, cherries, date fruit, fruit juices, eggplant, gooseberry, grape, grape juice, guava, jackfruit, mandarin, mango, mangosteen, mosambi (sweet lime) juice, honeydew melon, orange, orange juice, pears, pineapple, pomegranate, pomelo, satsuma, starfruits, strawberry, tomatoes, tangerine and watermelon. Therefore, this study would be extremely beneficial to the fruit cultivators, agricultural scientists, researchers, students, farmers, fruit processing industry, consumers and regulatory agencies, as it provides all the relevant published literature at one place.

\section{ANN IN FRUITS}

ANN has been applied in almost every aspect of agricultural science over past two decades, yet most applications are in the development stage. ANNs are useful tools for food safety and quality analyses, which include modeling of microbial growth, and from this predicting food safety; interpreting spectroscopic data, predicting physical, chemical, functional and sensory properties of various agricultural products during processing, storage and distribution. ANNs hold a great deal of promise for modeling complex tasks in process control and simulation and in applications of machine perception including machine vision and electronic nose for food safety and quality control [7]. The application of ANN for predicting the shelf life of food products in 
food industry is quite a new and effective approach. The ANN technique to dairy products is applied mainly because the shelf life evaluation in the laboratory is very cumbersome, expensive, and time-consuming, while the ANN procedure is sensitive, reliable, fast, simple and low-cost method for monitoring the authenticity of the products, which provide consumers with a safer food supply [8]. The published literature for various fruits using ANN modeling is presented below:

\section{A. Apple}

ANN modeling and several mathematical models were applied to predict the moisture ratio in an apple drying process by investigators. Four drying mathematical models were fitted to the data obtained from eight drying runs and the most accurate model was selected by researchers. Two sets of ANN modeling were also performed. In the first set, the data obtained from each pilot were modeled individually to compare the ANN predictions with the best mathematical model. In the second set of ANN modeling, the simultaneous effect of all the four input parameters including air velocity, air temperature, the thickness of apple slices and drying time was investigated. The results showed that the ANN predictions were more accurate in comparison with the best fitted mathematical model. In addition, none of the mathematical models was able to predict the effect of the four input parameters simultaneously, while the presented ANN model predicted this effect with a good precision [9].

\section{B. Apple juice}

Freeze-drying of foamed and non foamed apple juice was studied by Raharitsifa and Ratti [10] in order to assess if there is a reduction in process time due to foaming. Foams were prepared by whipping apple juice with methylcellulose or egg albumin at different concentrations. Foamed and non foamed juice samples having different thickness and different initial weight were frozen at $-40^{\circ} \mathrm{C}$, and then freeze dried at $20^{\circ} \mathrm{C}$ during $48 \mathrm{~h}$ under vacuum. Sample weight loss and temperature were followed at different process times. A mathematical model based on ANN was developed to represent foam kinetics and temperature curves during freeze-drying. This study revealed that freeze-drying of foamed materials is limited by heat transfer, while for non foamed ones, by mass transfer. It was shown that the insulation property characteristic of foams was more significant in slowing down the freeze-drying process than the increased surface area available for mass transfer due to foaming. ANN can be used to obtain excellent predictions of moisture content and temperature during the freeze-drying process.

\section{Avocados}

Maftoonazad et al. [11] applied ANN and hyperspectral techniques to model quality changes in avocados during storage at different temperatures. Avocados were coated using a pectin-based emulsion and stored at different temperatures $\left(10,15,20^{\circ} \mathrm{C}\right)$, along with uncoated control samples. At different time intervals during storage period, respiration rate, total color difference, texture and weight loss of samples were measured as conventional quality parameters. They used hyperspectral imaging to evaluate spectral properties of avocados. Multilayer ANNs were used in two ways to develop models for predicting quality parameters during storage. In the first set, ANN models were developed based on principal components of hyperspectral data as well as storage temperature and time. The optimal configuration of ANN model was obtained by varying the different model parameters. Results indicated ANN models to be accurate and versatile and they predicted the quality changes in avocado fruits better than the conventional regression models. Furthermore, the storage time-temperaturebased ANN models were better than the hyperspectrabased ANN models.

\section{Apricot}

In a study, Parpinello and others [12] used an array of 10 metal-oxide sensors, a so-called electronic nose, in combination with ANN to analyze the headspace of apricot fruits in order to classify 10 different cultivars. The ANN coupled to the electronic nose required a small computational effort to assure a satisfactory effectiveness. They explored different configurations, ranging from one to three hidden layers. The single hidden layer ANN with 35 neurons gave a correlation index higher than $80 \%$ on test data set. The trained system allowed at least $90 \%$ correct classification of apricot cultivars, showing the potential of these new tools in the quality control of fruits.

\section{E. Banana}

An electronic nose based system, which employs an array of inexpensive commercial tin-oxide odour sensors, was developed by Llobet and co-workers [13] to analyze the state of ripeness of bananas. Readings were taken from the headspace of three sets of bananas during ripening over a period of 8-14 days. Principal components analysis (PCA) and investigatory techniques were used to define seven distinct regions in multisensor space according to the state of ripeness of the bananas, predicted from a classification of bananaskin colours. Then three supervised classifiers, namely Fuzzy ARTMAP, LVQ and MLP were employed to classify the samples into the observed seven states of ripeness. It was found that the Fuzzy ARTMAP and LVQ classifiers outperformed the MLP classifier, with accuracies of $90.3 \%$ and $92 \%$, respectively, compared to MLP classifier (83.4\%). Furthermore, these methods were able to predict accurately the state of ripeness of unknown sets of bananas with almost same accuracy, i.e., $90 \%$. Finally, the workers observed that the Fuzzy 
ARTMAP classifier, unlike LVQ and MLP, was able to perform efficiently on-line learning in this application without forgetting previously learnt knowledge. All of these characteristics make the Fuzzy-ARTMAP-based electronic nose a very attractive instrument for determining non-destructively the state of ripeness of fruit.

\section{F. Blackberry}

The Andean blackberry is an important source of income in hillside regions of Colombia. However, growers have little reliable information on the factors that affect the development and yield of the crop. Therefore, there is a dearth of information on how to effectively manage the crop. Site specific information recorded by small-scale producers of the Andean blackberry on their production systems and soils coupled with publicly available meteorological data was used to develop models of such production systems. Multilayer perceptrons and Self-Organizing Maps were used by workers as computational models in the identification and visualization of the most important variables for modeling the production of Andean blackberry. ANNs were trained with information from 20 sites in Colombia, where the Andean blackberry is cultivated. Multilayer perceptrons predicted with a reasonable degree of accuracy the production response of the crop. The information obtained from ANN modeling can be used to determine sites that are suitable for Andean blackberry production, and to transfer of management practices from sites of high productivity to sites with similar environmental conditions, which currently have lower levels of productivity [14].

\section{G. Blackcurrant}

An ANN model for predicting flavour intensity in blackcurrant concentrates was investigated by Boccorh and Paterson [15]. Sets of ANN were developed from gas chromatographic data on flavour components (37) in 133 sorbent extracts from blackcurrant concentrates varying in season, geographical origin and processing technology. Investigators collected sensory data using ratio scaling on flavour intensities in drinks from concentrates. Relationships between chromatographic and sensory data for concentrates of three seasons (1989, 1990 and 1992) were modeled by ANNs with backpropagation using PCR scores as input. Predictions were compared with a global model from random concentrates from all three seasons. In predicting overall flavour intensity, ANN models were better fitted than partial least square (PLS) regression. Ability of ANN to simulate non-linear relationships observed in human perceptions could explain such improvements.

\section{H. Blueberries}

Chen and others [16] used ANN modeling for predicting quality changes during osmo-convective drying of blueberries for process optimization. Osmotic drying usually involves treatment of fruits in an osmotic solution of pre-determined concentration, temperature and time, and generally affects several associated quality factors such as colour, texture, rehydration ratio as well as the finish drying time in a subsequent drier (usually air drying). Multilayer ANN models with 3 inputs (concentration, osmotic temperature and contact time) were developed by analysts to predict 5 outputs: air drying time, color, texture, and rehydration ratio as well as a defined comprehensive index. The optimal configuration of ANN model was obtained by varying the main parameters of ANN: transfer function, learning rule, number of neurons and layers, and learning runs. The predictability of ANN models was compared with that of multiple regression model (MLR), confirming that ANN models had much better performance than conventional mathematical models. The prediction matrices and corresponding response curves for main processing properties under various osmotic dehydration conditions were used for searching the optimal processing conditions. Their results indicated that it is feasible to use ANN for prediction and optimization of osmo-convective drying for blueberries.

\section{Cherries}

A machine vision system was created to identify different types of tissue characteristics on cherries. It consisted of an enhanced NIR range vidicon black and white camera (sensing range 400-2000 nm), a monochrometer controlled light source, and a computer. Multiple spectral images of cherry samples were collected over the 680-1280 nm range at increments of $40 \mathrm{~nm}$. Using the spectral signatures of different tissues on cherry images, ANN modeling was implemented to pixel-wise classification. An enhanced genetic algorithm was applied to design the topology and evolve the weights for multilayer feedforward ANN. An average of $73 \%$ classification accuracy was achieved for correct identification as well as quantification of all types of cherry defects [17].

\section{J. Date fruit}

The kingdom of Saudi Arabia is the world's largest producer of date fruit. It produces almost 400 date varieties in bulk. During the harvesting season, the date grading and sorting pose problems for date growers. Since it is a labor intensive and time consuming process, it delays the post harvesting operations, which costs them dearly. The date grading and sorting is a repetitive process. In practice, it is carried out by humans manually through visual inspection. The manual inspection poses further problems in maintaining consistency in grading and uniformity in sorting. To speed up the process as well as maintain the consistency and uniformity, a prototypical computer vision based date grading and sorting system was designed and implemented. External quality features were defined. 
The system uses Red-Green-Blue images of the date fruits. From these images, it automatically extracts the aforementioned external date quality features. Based on the extracted features, it classifies dates into three quality categories (grades 1, 2 and 3) defined by the experts. Performance of ANN classifier was studied and accuracy of the system on pre-selected date samples was tested. The test results showed that the system can sort $80 \%$ dates accurately [18].

\section{K. Eggplant}

Saito et al. [19] proposed an eggplant grading system using image processing and ANN classification. They used the lighting conditions for taking color components of the eggplant image effectively with shape parameters such as length, girth, etc. using image processing. On the other hand, bruises of the eggplants were detected and classified based on the color information by using ANN classification.

\section{Fruit juices}

Rai and co-workers [20] developed an ANN model for the prediction of viscosity of fruit juice as a function of concentration and temperature. The fruit juices considered by them were orange, peach, pear, malus floribunda and black current. The viscosity data of juices (1.53-3300mPas) were obtained from the literature for a wide range of concentration $\left(5-70{ }^{\circ}\right.$ Brix) and temperature $\left(30.7-71.7^{\circ} \mathrm{C}\right)$. Several configurations were evaluated while developing the optimal ANN model. The optimal ANN model consisted of two hidden layers with two neurons in each hidden layer. This model was able to predict viscosity with a MAE of $3.78 \mathrm{mPas}$. The performance of the ANN was checked using experimental data. Predicted viscosity using the ANN proved to be a simple, convenient and accurate method. They concluded that the model can be incorporated in the heat transfer calculations during fruit processing where concentration and temperature dependent viscosity values are required. This may also be useful in mass transfer calculations during filtration of the juice using membranes for clarification.

\section{Gooseberry}

Based on the study of gooseberry's dielectric properties, Guo and Zhang [21] made use of the ANN to classify different kinds of gooseberries. They indicated that the crucial point of this classification is getting the characteristic parameters. If characteristic parameters of gooseberries are obtained and a suitable ANN is chosen, then gooseberry's can be classified with lots of training.

\section{N. Grape}

Janik et al. [22] conducted a study to compare the performance of partial least squares (PLS) regression analysis and ANN for the prediction of total anthocyanin concentration in red-grape homogenates from their visible-near-infrared (Vis-NIR) spectra. The PLS prediction of anthocyanin concentrations for new-season samples from Vis-NIR spectra was characterized by regression non-linearity and prediction bias. In practice, this usually requires the inclusion of some samples from the new vintage to improve the prediction. The use of WinISI LOCAL partly alleviated these problems but still resulted in increased error at high and low extremes of the anthocyanin concentration range. ANN regression was investigated as an alternative method to PLS due to the inherent advantages of ANN for modeling non-linear systems. The proposed method combines the advantages of the data reduction capabilities of PLS regression with the non-linear modeling capabilities of ANN. With the use of PLS scores as inputs for ANN regression, the model was shown to be quicker and easier to train than using raw full-spectrum data. The ANN calibration for prediction of new vintage grape data using PLS scores as inputs was more linear and accurate than global and LOCAL PLS models, and appeared to reduce the need for refreshing the calibration with new-season samples. ANN with PLS scores required fewer inputs and was less prone to overfitting than using PCA scores. Researchers concluded that the variation of ANN method, using carefully selected spectral frequencies as inputs, resulted in prediction accuracy comparable to those using PLS scores, but as for PCA inputs, was also prone to overfitting with redundant wavelengths.

\section{O. Grape juice}

Mateo and others [23] studied Multi Layer Perceptron (MLP) and Radial Basis function networks to predict ochratoxin $A$ concentration over time in grape-based cultures of aspergillus carbonarius under different conditions of temperature, water activity $\left(\mathrm{a}_{\mathrm{w}}\right)$ and subinhibitory doses of the fungicide carbendazim. The results obtained by them established ANNs as useful tools, and should be fully explored in the field of food safety.

\section{P. Guava}

The respiration rate prediction of fresh produce is crucial for designing and operating postharvest storage systems. The investigators constructed and evaluated respiration models of guava fruit by using not only the enzyme, chemical kinetics, but also ANN with the experimental data obtained from 5, 10, 15, 20, 25 and $30{ }^{\circ} \mathrm{C}$ (for constructing) as well as 12 and $22{ }^{\circ} \mathrm{C}$ (for evaluating) by closed system method. All of the developed models showed good agreement with actual observations. As regards fidelity, the ANN model with topologic structure of $3 \times 9 \times 1$ trained by the LevenbergMarquardt algorithm, evaluation results were such that the mean absolute percentage error (MAPE) and the two-tailed Pearson correlation coefficient (r) were 5.31 and 0.997 for $12{ }^{\circ} \mathrm{C}, 4.85$ and 0.995 for $22{ }^{\circ} \mathrm{C}$, had superiority over the two other models. The results 
indicated that the ANN approach is a more precise method, and can be used for predicting the respiration rate of guava fruit [24].

\section{Q. Jackfruit}

Bala et al. [25] predicted the performance of a solar tunnel drier for drying jackfruit bulbs and leather. They developed a multi-layered ANN model to predict the performance of the solar tunnel drier. Using solar drying data of jackfruit bulbs and leather, the model was trained using backpropagation algorithm. The prediction performance of the drier was found to be excellent after it was adequately trained. The results showed that ANN can be used for predicting the potential of the solar tunnel drier for drying jackfruit bulbs and leather at different locations.

\section{R. Mandarin}

A portable near infrared spectroscopy system was developed for assessing the quality of Nanfeng mandarin fruit. One hundred and fifty-three Nanfeng mandarin samples were used to measure the performance of the system. Several pre-treatment methods were adopted to process the spectra. Then Support Vector Machine (SVM), Back Propagation Neural Network (BPNN) and PLS were used to build models for soluble solids content (SSC), titratable acidity (TA), vitamin $\mathrm{C}$ and surface color. The best results were obtained by SVM. The correlation coefficient $\left(\mathrm{R}^{2}\right)$ and root mean square error (RMSE) of prediction were $\left(0.93,0.65^{\circ} \mathrm{Brix}\right),(0.66$, $0.09 \%),(0.81,2.7 \mathrm{mg} / 100 \mathrm{~g})$ and $(0.57,0.81)$ for SSC, $\mathrm{TA}$, vitamin $\mathrm{C}$ and color, respectively. The results demonstrated that the portable near infrared spectroscopy was feasible for determining the Nanfeng mandarin quality non-destructively [26].

\section{S. Mango}

A predictive model for heat and mass transfer using ANN was proposed in order to obtain on-line predictions of temperature and moisture kinetics during the drying of mango [27]. The model takes into account shrinkage of the product as a function of moisture content. Two separate feedforward networks with one hidden layer were used. The best fitting with the training dataset was obtained with three neurons in the hidden layer, which made possible to predict heat and mass transfer with accuracy, at least as good as the experimental error, over the whole experimental range. On the validation dataset, simulations and experimental kinetics test were in good agreement. The researchers recommended that the developed model can be used for on-line state estimation and control of drying processes.

\section{T. Mangosteen}

In another published research, multilayer ANN with backpropagation algorithm were applied for predicting mangosteen quality during storage at the most appropriate pre-storage conditions, which performed the longest storage period. Each condition was a combination of three pre-storage treatments involving pre-cooling at $20^{\circ} \mathrm{C}$, bee waxing concentration of $6 \%$ and strecth film single wrapping. To decide, which combination gave best fruit quality on their prolonged storage, it was investigated by panelist preference by using Hedonic scale. Based on the experiment, precooling at $20^{\circ} \mathrm{C}$ followed by bee waxing concentration of $6 \%$ and strecth film single wrapping provided minimum quality changes over $40^{\circ} \mathrm{C}$. The successfully trained model constituted network architecture of 30,000 iterations; 14 nodes in hidden layer; learning rate constant 0.8 and momentum 0.7 . It was represented by $\mathrm{R}^{2}$ close to 1 (more than 0.99 ) for each parameter, indicating that the model was good to memorize data [28].

The optimum conditions of extraction time, solid to solvent ratio, and methanol concentration for extracting total phenolic content (TPC) from mangosteen hull powder were investigated using RSM and ANN methodologies. The experimental data obtained was adequately fitted into second-order polynomial models with $\mathrm{R}^{2} 0.897$. Response surface analysis showed that the optimal extraction parameters, which gave a maximum TPC yield of $140.66 \mathrm{mg}$ gallic acid equivalent (GAE)/g powder were from a $2 \mathrm{~h}$ extraction with 0.05 solid to solvent ratio and at $69.77 \%$ methanol concentration. Analysis using ANN predicted data, which showed a higher $\mathrm{R}^{2}$ value of 0.945 and average absolute deviation (ADD) values of $4.01 \%$ versus $5.37 \%$ for the RSM. The results suggested that ANN is a better modeling technique for non-linear data for predicting TPC extracted from mangosteen hull powder compared to the RSM [29].

\section{U. Mosambi (Sweet Lime) juice}

Rai and others [30] used ANN to describe the permeate flux and permeate concentration (total soluble solid) profiles during the ultrafiltration of synthetic fruit juice and mosambi (sweet lime) juice dynamically. They predicted the permeate flux and total soluble solid of permeate as a function of transmembrane pressure, sucrose, pectin concentration in the feed and the processing time. A multilayer feedforward network structure with input, output and hidden layers was created along with the backpropagation algorithm for training of ANN models. Two neural network models were constructed to predict the permeate flux and the total soluble solids in the permeate using the filtration data of the synthetic juice. The modeling results showed that there was an agreement between the experimental data and predicted values, with mean absolute errors (MAE) less than $1 \%$ of the experimental data. Also, the trained network was able to capture accurately the nonlinear dynamics of synthetic fruit juice and the actual mosambi (sweet lime) juice even for a new condition that has not been used in the training process. 


\section{Honeydew melon}

Thai et al. [31] evaluated the performance of neural networks in the evaluation of human preferences for honeydew melon.

\section{W. Orange}

ANNs are among the most popular techniques for non-linear multivariate calibration in complicated mixtures using spectrophotometric data. In this study, Fe and $\mathrm{Ni}$ simultaneously determined aqueous medium with xylenol orange $(\mathrm{XO})$ at $\mathrm{pH}$ 4.0. In this way, after reducing the number of spectral data using PCA, an ANN consisting of three layers of nodes was trained by applying a backpropagation learning rule. Sigmoid transfer functions were used in the hidden and output layers to facilitate non-linear calibration. The ANN method gave satisfactory results [32].

\section{Orange juice}

The effects of feed flow rate, inlet-air temperature, and atomizer speed in an orange juice semi-industrial spray dryer were studied on seven performance indices, namely: residual moisture content of orange juice powder, particles size, bulk density, average time of wettability, insoluble solids, outlet air temperature and dryer yield. A supervised ANN trained by back propagation algorithm was developed to predict seven performance indices based on the three input variables. The numbers of patterns used in this study were 80, used for training, verification, and testing the ANN. After evaluating a large number of trials with various ANN architectures, the optimal model was a four-layered backpropagation ANN, with 14 and 10 neurons in the first and the second hidden layers, respectively. The ANN technology was found to be a useful tool to investigate, approximate and predict the physical properties of orange juice powder as well as process parameters of spray dryers [33].

\section{Y. Pears}

A new and simple algorithm to judge the presence and integrity of stems on Huanghua pears and a method to describe the irregular shapes of the pears were developed using Fourier transformation and ANN modeling. A backpropagation feedforward ANN was used to identify the shape of Huanghua pears. By applying the Fourier descriptor in combination with the ANN, a classification accuracy of $90 \%$ was achieved [34].

Another study was carried out on pears by Zhou and $\mathrm{Li}$ [35] on texture analysis of MR image for predicting the firmness of Huanghua pears during storage using an ANN model. Firmness, a main index of quality changes, is important for the quality evaluation of fruits. Seven co-occurrence matrix-derived texture analysis (TA) variables and one run-length matrix $\mathrm{TA}$ variable significantly correlated with firmness were considered as inputs to the ANN. Several ANN models were evaluated when developing the optimal topology. The optimal ANN model consisted of one hidden layer with 17 neurons in the hidden layer. This model was able to predict the firmness of the pears with a MAE of $0.539 \mathrm{~N}$ and $\mathrm{R}^{2}=0.969$. The results established the potential of TA parameters of MR images combined with ANN for investigating the internal quality characteristics of fruits during storage.

\section{Z. Pineapple}

Boonmung et al. [36] planned a study for evaluating performance of ANN modeling for pineapple grading. The main objective of their experiment was to evaluate resonant frequency, firmness and soluble solids for pineapple classification using ANN as the analytical tool. A sample of 149 pineapples was classified based on their internal qualities into five classes: unripe, partially ripe, ripe, partially overripe and completely overripe. The proposed ANN model successfully classified pineapples into merely three classes as unripe, ripe and completely overripe. The most effective model was obtained when both resonant frequency and soluble solids were included in the model. The classification accuracy was more than $83 \%$ for all three classes.

\section{AA. Pomegranate}

Drying of pomegranate arils was predicted by Motevali and co-workers [37] by creating ANN and mathematical models. Ten semi-theoretical and empirical models were fitted to the experimental data to evaluate and select the best model for thin-layer drying of pomegranate arils. Experiments were conducted at six temperature levels of $45,50,55,60,65$ and $70^{\circ} \mathrm{C}$, and three levels of air velocity $(0.5,1$ and $1.5 \mathrm{~m} / \mathrm{s})$. Microwave pre-treatments were used for samples and the results were compared with those of control (no pretreatments). Regression analysis of mathematical models showed that the Midili model fitted best to the measured data. However, regarding $\mathrm{R}^{2}$ and MSE criteria, ANN modeling yielded a better prediction of pomegranate arils moisture ratio during drying of arils compared to all the mathematical models studied.

Youssefi et al. [38] compared ANN and Response Surface Methodology (RSM) in the prediction of quality parameters of spray-dried pomegranate juice. RSM is a frequently used method for empirical modeling and prediction in the processing of biological media. The ANN has recently grown to be one of the most efficient methods for empirical modeling and prediction, especially for non-linear systems. In their investigation, the effects of the carrier type, carrier concentration, and concentration of crystalline cellulose in a pomegranate juice spray-drying process were analyzed on five quality parameters - drying yield, solubility, color change, total anthocyanin content, and antioxidant activity-using RSM and ANN methods. A central composite rotatable 
experimental design (CCRD) and a feedforward multilayered perceptron ANN trained using backpropagation algorithms for three independent variables were developed to predict the five outputs. The final selected ANN model (3-10-8-5) was compared to the RSM model for its modeling and predictive abilities. The predictive abilities of both the ANN and RSM were compared using a separate dataset of 18 unseen experiments based on RMSE, MAE, and $\mathrm{R}^{2}$ for each output parameter. The results confirmed the superiority of a properly trained ANN in capturing the non-linear behavior of the system and the simultaneous prediction of five outputs.

\section{BB. Pomelo}

Single factor tests and orthogonal experiments were conducted [39] to acquire optimum extraction craft of flavonoids from pomelo peel. The alcohol concentration, solvent amount, extraction temperature and extraction time were considered to be influencing factors. The results of 20 single tests and 9 orthogonal experiments were used as training samples to design and train a 4-5-1 three-layered backpropagation ANN. Then the 625 designed extraction conditions were predicted by the terminative ANN model; and 3 combinations of extraction conditions, which could bring about high yield of flavonoids were acquired. The results showed that the craft acquired from ANN could obtain 5.4\% more flavonoids than that of orthogonal experiments.

\section{Satsuma}

An optimal watering scheduling that improves the quality of Satsuma mandarins grown in the field was investigated using ANN and genetic algorithms. Fruit responses and climate factors were determined monthly from August to November, 1996-2004. Dynamic changes in the sugar and citric acid contents of the Satsuma mandarins, as affected by the rainfall and sunshine duration, were first identified using ANN, and then an optimal watering scheduling (rainfall management) that maximizes the sugar content and minimizes the citric acid of the Satsuma mandarins was determined through simulation of the identified ANN model using genetic algorithms [40].

Although most of the process of canning mandarin segments is already automated, this has still not been achieved with the on-line inspection and sorting of the fruit because of the difficulty in the handling of the product and the complexity of the inspection software required classifying the segments following subjective criteria. A machine vision-based system has been created to classify the objects that reach the line into four categories, detecting broken fruit, attending basically to the shape of the fruit. A full working prototype has been developed for singulating, inspecting and sorting Satsuma (Citrus unshiu) segments. The segments are transported over semi-transparent conveyor belts to allow illuminating the fruit from the bottom to enhance the shape of the segments against the background. The system acquires images of the segments using two cameras connected to a single computer and processes them in less than $50 \mathrm{~ms}$. By extracting morphological features from the objects, the system automatically identifies pieces of skin and other raw material, and separates whole segments from broken ones. It is also capable to grade between those with a slight or a large degree of breakage. Tests showed that the machine vision neural network modeling was able to correctly classify $93.2 \%$ of sound segments [41].

\section{DD. Starfruits}

Software for detecting the quality features of golden delicious starfruits of Averrhoa carambola L. genus were developed for automated inspection system using machine vision technology. The features considered were colour and shape. The use of artificial classifiers such as linear discrimination analysis and MLP-ANN as a tool to detect starfruit maturities such as unripe, under ripe, ripe and overripe in HSI colour space were investigated. The colour spectra of matured and unmatured fruits were characterized using all colour features ranging from hue 10 to hue 74 , using principal hues generated by Wilks-lambda analysis. Experiments performed on 200 independent starfruit samples revealed that linear discriminant analysis after Wilks-lambda analysis was more precise in classification than direct application of linear discriminant analysis. However, the classification accuracy of multi-layer perceptron remained relatively the same before and after feature reduction. Overall, the average correct classification for linear discriminant analysis and multi-layer perceptron was $95.3 \%$ and $90.5 \%$, respectively during testing stage. Meanwhile, the use of Fourier transform was investigated for shape discrimination. This algorithm produced $100 \%$ success rate in detecting starfruits by three shape categories: well-formed, slightly deformed and seriously deformed. Both colour and shape analysis were easily affected by the variation of lighting levels, and this contributed to the major classification error [42]

\section{EE. Strawberry}

In order to provide an efficient and running analytical tool to strawberry plant breeders who have to characterize and compare the aromatic properties of new cultivars to those already known, a HS-SPME/GC-MS analysis method has been coupled with a statistical treatment method issued from the current development of ANN, and more specifically, the unsupervised learning systems called Kohonen self-organizing maps (SOMs) [43]. Seventy strawberry samples harvested at CIREF from 17 known varieties were extracted by using a DVB/Carboxen/PDMS SPME fiber according to the headspace procedure, and then chromatographed. Panels of 23 characteristic aromatic constituents were selected according to the published results relative to strawberry aroma. The complex resulting matrix, collecting the 
relative abundance of the 23 selected constituents for each sample were used as inputs, into the SOM software adapted and optimized from the Kohonen approach. After a period of training, the self-organized system afforded a map of virtual strawberries to which real samples were compared and plotted in the best matching unit (BMU) of the map. The efficiency for discriminating the real samples according to their variety is dependent on the number of units selected to define the map. In this case, a 24-unit map allowed the complete discrimination of the 17 selected varieties. Moreover, to test the validity of this approach, two additional samples were blind-analyzed and the results were computed according to the same procedure. At the end of this treatment, both samples were plotted into the same unit as those of the same variety used for training the map. Results from ANN modeling were found to be satisfactory.

In another study, the SPME-GC-MS method (chemical analysis) coupled with KSOM-ANN treatment of the results (statistical algorithm) has proved to be efficient to classify 70 strawberry samples harvested in the same year, through the 17 varieties to which they belonged, in a two-dimensional map. The study confirmed that the results were not dependent on the year of strawberry production and highlights what effects were observed between results obtained in different years. Samples of different strawberry varieties were harvested during the three campaigns of 2000, 2001, and 2002 and analyzed independently. The chemical data matrix obtained in each case allowed the verification of the proposal that the same discriminative effect could be obtained independently of the year of production by using maps of different sizes. Therefore, 30 measures obtained from samples of 9 varieties in 2000, 54 measures from 13 varieties in 2001, and 80 measures from 20 varieties in 2002 were correctly classified by using 20,35, and 56 hexagon maps, respectively. In a second analysis based on the 2002 production, the chemical differences between variety aromatic features were noted through the increasing size of the map used. Finally, results relative to 7 varieties cultivated in $2001 \& 2002$, and stored under exactly the same conditions were computed together for elaborating a single map. An interesting effect of double classification according to the year and the varieties was observed [44].

\section{FF. Tomatoes}

Movagharnejad and Nikzad [45] performed experiments on drying of tomatoes in a tray dryer covering different variables like power of heater and air flow velocity. They modeled the data using ANN and empirical mathematical equations. The results were compared with experimental data, and it was found that the predictions of the ANN model fit the experimental data more accurately in comparison to the various mathematical equations. Wang et al. [46] developed a method to spectrally predict late blight infections on tomatoes based on ANN. The ANN was designed as a backpropagation (BP) neural network that used gradient - descent learning algorithm. Through comparing different network structures, they selected a $3-25$ - 9 1 network structure. Two experimental samples from field experiments and remotely sensed image data sets were used to train the ANN to predict healthy and diseased tomato canopies with various infection stages for any given spectral wavelength $(\mu \mathrm{m})$ intervals. Results of discrete data indicated different levels of disease infestations. The correlation coefficients of prediction values and observed data were 0.99 and 0.82 for field data and remote sensing image data, respectively. In addition, they predicted the field data based on the remote sensing image data and predicted the remote sensing image data with field data using the same network structure, and the results showed that $\mathrm{R}^{2}$ was 0.62 and 0.66 , respectively, suggesting that ANN with backpropagation training could be used in spectral prediction.

\section{GG. Tangerine}

Boonprasom and Bumroongitt [47] applied ANN to forecast the tangerine yield, taking into account some influential factors, especially the weather conditions. They collected data and information relating to tangerine yield of Chiang Mai Province from 1992 to 2001. Weather data such as average monthly rainfall and average monthly temperature of relevant periods were also obtained by them. They created 5 ANN models with different input factors or different structures for three sections of the experiments. The results indicated that the ANN had high potential and ability to forecast tangerine yield accurately despite small set of data available. The amount of rainfall was observed to have strong influence and contributed important information to the ANN in forecasting tangerine yield.

Rithmanee and co-workers [48] predicted quality of tangerine after truck transportation from the packing house in Chiangmai to the wholesale market in Bangkok, as indexed by changes in vitamin $\mathrm{C}$, titratable acidity (TA), $\mathrm{pH}$, total soluble solids (TSS), TSS/TA; weight loss percentage, decay percentage, mechanical damage percentage and surface color change. The statistic treatment structure for the experiment was a 32 factorial design in RCBD with three replications (trips). Temperature and relative humidity of the fruits were taken into the model as covariates. ANN was used as a tool to predict postharvest quality and losses, then compared the results with those using MLR. From the 27 data records, 22 data records were used for training set and 5 data records for testing set to predict quality of tangerine. ANN showed its potential and ability to predict tangerine after truck transportation quite accurately; the values of error were observed to be lower and $\mathrm{R}^{2}$ higher than MLR. The RMSE of the prediction by several ANN models ranged from 0.014 to 0.911 , and $\mathrm{R}^{2}$ ranged from 0.634 to 0.942 . RMSE from prediction using MLR models ranged from 0.044 to 5.823 , and $\mathrm{R}^{2}$ 
ranged from 0.120 to 0.671 .

\section{HH. Watermelon}

Baki and others [49] created a non-destructive watermelon classification method using Mel-Frequency Cepstrum Coefficients (MFCC) and MLP neural network. Acoustic signals were collected from thumping noises of ripe and unripe watermelon fruits. MFCC was then used to convert the signals into MFCC coefficients. The coefficients were used to train a MLP, and the MLP gave the final decision on the watermelon ripeness state. The results showed that the proposed method was able to discriminate between ripe and unripe watermelons with $77.25 \%$ accuracy.

\section{CONCLUSION}

This comprehensive review of the published literature concerning application of artificial neural network (ANN) technologies for predictive assessment in fruits showed consistent increase in its use over the past decade. This communication includes a wide range of fruits, viz., apple, apple juice, avocado, apricot, banana, blackberry, blackcurrant, blueberries, cherries, date fruit, fruit juices, eggplant, gooseberry, grape, grape juice, guava, jackfruit, mandarin, mango, mangosteen, mosambi (sweet lime) juice, honeydew melon, orange, orange juice, pears, pineapple, pomegranate, pomelo, satsuma, starfruits, strawberry, tomatoes, tangerine and watermelon. The literature search revealed that still a lot of research needs to be done using ANN in the area of fruit cultivation, processing, packaging, storage and transportation. ANN is a simple and accurate modeling method that has been applied in all areas of science and technology. The author hopes that in future more and more concerned scientists would use these non-linear modeling tools (ANNs) for predictive assessment in fruits, as it is evident from the analysis of this paper that research related to fruits using ANN has picked up. The purpose of writing this review paper was to present all published work related to fruits using ANN at one place, which would be very beneficial for farmers engaged in the cultivation of fruits, food processing industry, scientists, agricultural technologists and students.

\section{REFERENCES}

[1] Y. Huang, L.J. Kangas and B.A. Rasco, "Applications of artificial neural networks (ANNs) in food science," Crit. Rev Food Sci. Nutr., Vol. 47, No.2, pp.133-126, 2007.

[2] Sumit Goyal, "Artificial neural networks in vegetables: A comprehensive review," Sci. J. of Crop Sci., Vol.2, No.7, pp. 75-94, 2013.

[3] Sumit Goyal, "Artificial neural networks (ANNs) in food science - A review," Int. J. of Sci. World, Vol.1, No.2, pp.19-28, 2013.

[4] Sumit Goyal, "Predicting properties of cereals using artificial neural networks: A review," Sci. J. of Crop Sci., Vol.2, No.7, pp. 95-115, 2013.

[5] Sumit Goyal and G.K. Goyal, "Machine learning cascade algorithm for analyzing shelf life of processed cheese," VAWKUM Trans. on Comput. Sci., Vol.2, No.1, pp.1-6, 2013.

[6] G.K Goyal and Sumit Goyal, "Cascade artificial neural network models for predicting shelf life of processed cheese," J. of Adv. in Info. Tech., Vol.4, No.2, pp.80-83, 2013.

[7] M. Bhotmange and P. Shastri, “(Eds.) Application of artificial neural networks to food and fermentation technology," ANN - Indus. and Con. Eng. App., Prof. Kenji Suzuki, ISBN: 978-953-307220-3, InTech, 2011.

[8] Sumit Goyal and G.K. Goyal, “. Artificial neural networks for dairy industry: A Review," J. of Adv. Comp. Sci. \& Tech. Vol.1, No.3, pp.101-115, 2012.

[9] A. Khoshhal, A.A. Dakhel, A. Etemadi and S. Zereshki, "Artificial neural network modeling of apple drying process," J. of Food Process Eng., Vol.33, pp.298-313, 2010.

[10] N. Raharitsifa and C. Ratti, "Foam-mat freezedrying of apple juice part 1: Experimental data and ANN simulations," J. of Food Process Eng. Vol.33, pp.268-283, 2010.

[11] N. Maftoonazad, Y. Karimi, H.S. Ramaswamy and S.O. Prasher,“ Artificial neural network modeling of hyperspectral radiometric data for quality changes associated with avocados during storage," J. of Food Process. and Preserv., Vol.35, No.4, pp.432-446, 2011.

[12] G.P. Parpinello, A. Fabbri, S. Domenichelli, V. Mesisca, L. Cavicchi and A. Versari, "Discrimination of apricot cultivars by gas multisensor array using an artificial neural network," Biosys. Eng., Vol. 97, No.3, pp.371-378, 2007.

[13] E. Llobet, E.L. Hines, J.W. Gardner and S. Franco, "Non-destructive banana ripeness determination using a neural network-based electronic nose," Measurement Sci. and Tech., Vol.10, No.6, pp.538548, 1999.

[14] D. Jiménez, J. Cock, H.F. Satizábal, M.A. Barreto, A. Pérez-Uribe, A. Jarvis and P.V. Damme, “Analysis of Andean blackberry (Rubus glaucus) production models obtained by means of artificial neural networks exploiting information collected by small-scale growers in Colombia and publicly available meteorological data," Comput. and Electron. in Agric., Vol.69, No.2, pp.198-208, 2009.

[15] R.K. Boccorh and A. Paterson, "An artificial neural network model for predicting flavour intensity in blackcurrant concentrates," Food Qual. and Prefer., Vol.13, No.2, pp.117-128, 2002.

[16] C.R. Chen, H.S. Ramaswamy and I. Alli, "Prediction of quality changes during osmoconvective drying of blueberries using neural network models for process optimization," Drying 
Tech.: An Int. J., Vol.19, No.3-4, pp.507-523, 2001.

[17] D. Guyer and X. Yang, "Use of genetic artificial neural networks and spectral imaging for defect detection on cherries. Comput. and Electron. in Agric., Vol.29, No.3, pp.179-194, 2000.

[18] Y.A. Ohali, "Computer vision based date fruit grading system: Design and implementation," J. of King Saud Uni. - Comp. and Info. Sci., Vol.23, No.1, pp.29-36, 2011.

[19] Y. Saito, T. Hatanaka, K. Uosaki and K. Shigeto, "Eggplant classification using artificial neural network," Proc. of the Int. Joint Conf. on Neural Netw., Vol.2, pp.1013-1018, 2003.

[20] P. Rai, G.C. Majumdar, S. DasGupta and S. De, "Prediction of the viscosity of clarified fruit juice using artificial neural network: a combined effect of concentration and temperature," J. of Food Eng., Vol.68, pp.527-533, 2005.

[21] H.L. Guo and L.X. Zhang, “Application of artificial neural network for classification of gooseberry species," J. Agric. Mech. Res., Vol. 12, pp.195-198, 2006.

[22] L.J. Janik, D. Cozzolino, R. Dambergs, W. Cynkar and M. Gishen, "The prediction of total anthocyanin concentration in red-grape homogenates using visible-near-infrared spectroscopy and artificial neural networks," Analytica Chimica Acta., Vol.594, No.1, pp.107118, 2007.

[23] F. Mateo, R. Gadea, A'. Medina, R. Mateo and M. Jime'nez, "Predictive assessment of ochratoxin A accumulation in grape juice based-medium by Aspergillus carbonarius using neural networks," J. of App. Microbio., Vol. 107, pp.915-927, 2009.

[24] Z. Wang, H. Duan, and C. Hu, "Modelling the respiration rate of guava (Psidium guajava L.) fruit using enzyme kinetics, chemical kinetics and artificial neural network," Euro. Food Res. and Tech., Vol.229, No.3, pp.495-503, 2009.

[25] B.K. Bala, M.A. Ashraf, M.A. Uddin and S. Janjai, "Experimental and neural network prediction of the performance of a solar tunnel drier for drying jackfruit bulbs and leather," J. of Food Process Eng., Vol.28, No.6, pp.552-566, 2005.

[26] S. Xudong, Z. Hailiang and L. Yande, "Nondestructive assessment of quality of Nanfeng mandarin fruit by a portable near infrared spectroscopy." Int. J. of Agri. and Biological Eng., Vol.2, No.1, pp.65-71, 2009

[27] J.A. Hernández-Pérez, M.A. García-Alvarado, G. Trystram and B. Heyd, "Neural networks for the heat and mass transfer prediction during drying of cassava and mango," Innov. Food Sci. \& Emerging Tech., Vol. 5, No.1, pp.57-64, 2004.

[28] Sutrisno, I.M. Edris and Sugiyono, "Quality prediction of mangosteen during storage using artificial neural network," Int. Agric. Eng. Conf., Bangkok, Thailand, 7 - 10 December, 2009.

[29] C.Y. Cheok, N.L. Chin, Y.A. Yusof, R.A. Talib and C.L. Law, "Optimization of total phenolic content extracted from Garcinia mangostana Linn. hull using response surface methodology versus artificial neural network," Industrial Crops and Prod. Vol.40, pp.247-253, 2012.

[30] P. Rai, G.C. Majumdar, S. DasGupta and S. De, "Modeling the performance of batch ultrafiltration of synthetic fruit juice and mosambi juice using artificial neural network," J. of Food Eng., 71, 273281, 2005.

[31] C.N. Thai, A.V.A. Resurreccion, G.G. Dull and D.A. Smittlle, "Modeling consumer preferences with neural networks," Am. Soc. Agric. Eng. Pap. 907550. The Society: St. Joseph, MI, 1990.

[32] M. Kompany-Zareh, A. Massoumi and S. PezeshkZadeh, "Simultaneous spectrophotometric determination of $\mathrm{Fe}$ and $\mathrm{Ni}$ with xylenol orange using principal component analysis and artificial neural networks in some industrial samples," Talanta., Vol.48, No.2, pp.283-292, 1999.

[33] G.R. Chegini, J. Khazaei, B. Ghobadian and A.M. Goudarzi, "Prediction of process and product parameters in an orange juice spray dryer using artificial neural networks," J. of Food Eng. 84(4), 534-543, 2008.

[34] Y. Ying, H. Jing, Y. Tao and N. Zhang, "Detecting stem and shape of pears using fourier transformation and an artificial neural network," Am. Soc. of Agric. Eng., Vol.46, No.1, pp.157-162, 2003.

[35] R. Zhou and Y. Li, "Texture analysis of MR image for predicting the firmness of Huanghua pears (Pyrus pyrifolia Nakai, cv. Huanghua) during storage using an artificial neural network," Magnetic Resonance Imaging., Vol.25, No.5, pp.727-732, 2007.

[36] S. Boonmung, B. Chomtee and K. Kanlayasiri, "Evaluation of artificial neural networks for pineapple grading," J. of Texture Stu., Vol.37, No.5, pp.568-579, 2006.

[37] A. Motevali, S. Minaei, M.H. Khoshtaghaza, M. Kazemi and A.M. Nikbakht, "Drying of pomegranate arils: comparison of predictions from mathematical models and neural networks," Int. J. of Food Eng., Vol.6, No.3, pp.1-20, 2010.

[38] S. Youssefi, Z. Emam-Djomeh and S.M. Mousavi, "Comparison of artificial neural network (ANN) and response surface methodology (RSM) in the prediction of quality parameters of spray-dried pomegranate juice," Drying Tech.: An Int. J., Vol.27, No.(7-8), pp.910- 917, 2009.

[39] H. Huang and W.R. Li, "Application of artificial neural network (ANN) on the extraction of flavonoids from pomelo peel," Hubei Agric. Sci., Vol.10, pp. 048, 2011.

[40] T. Morimoto, Y. Ouchi, M. Shimizu and M.S. Baloch, "Dynamic optimization of watering Satsuma mandarin using neural networks and genetic algorithms," Agric. Water Manag., Vol.93, No.(1-2), pp. 1-10, 2007.

[41] J. Blasco, N. Aleixos, S. Cubero, J. Gómez-Sanchís 
and E. Moltó, "Automatic sorting of satsuma (Citrus unshiu) segments using computer vision and morphological features," Comput. and Electron. in Agric., Vol.66, No.1, pp.1-8, 2009.

[42] M.Z. Abdullah, J. Mohamad-Saleh, A.S. FathinulSyahir and B.M.N. Mohd-Azemi, "Discrimination and classification of fresh-cut starfruits (Averrhoa carambola L.) using automated machine vision system," J. of Food Eng., Vol.76, No.4, pp.506523, 2006.

[43] L. Urruty, J.L. Giraudel, S. Lek, P. Roudeillac and M. Montury, "Assessment of strawberry aroma through SPME/GC and ANN methods, classification and discrimination of varieties," J. of Agric. and Food Chem., Vol. 50, No.11, pp.31293136, 2002.

[44] V.D. Boishebert, L. Urruty, J.L. Giraudel and M. Montury, "Assessment of strawberry aroma through solid-phase microextraction-gas chromatography and artificial neuron network methods. Variety classification versus growing years," J. of Agric. and Food Chem., Vol.52, No.9, pp. 2472-2478, 2004.

[45] K. Movagharnejad and M. Nikzad, "Modeling of tomato drying using artificial neural network," Comput. and Electron. in Agric., Vol.59, pp. 78-85, 2007.

[46] X. Wang, M. Zhang, J. Zhu and S. Geng, "Spectral prediction of Phytophthora infestans infection on tomatoes using artificial neural network (ANN)," Int. J. of Remote Sensing., Vol.29, No.6, pp.16931706, 2008.

[47] P. Boonprasom and G. Bumroongitt, "Prediction of tangerine yield using artificial neural network
(ANN)," CMU J., Vol4, No.1, pp. 39-48, 2005.

[48] T. Rithmanee, G. Bumroonggit and P. Boonprasom, "Quality prediction of 'sai nam pung' tangerine after truck transportation using artificial neural network. Acta Horticulturae, Vol.802, pp.379-384, 2008.

[49] S. Baki, Z.M. Annuar, I.M. Yassin, A.H. Hasliza, A. Zabidi, "Non-destructive classification of watermelon ripeness using mel-frequency cepstrum coefficients and multilayer perceptrons," The IEEE Int. Joint Conf. on Neu. Netw., pp.1-6, 2010.

Sumit Goyal, received his Bachelor and Master's degree from the central university of Government of India. He has published research papers in many international journals throughout the world, which have been cited more than 550 times. Besides that, he has also written book chapters, instructional manuals, marketing collaterals, user manuals, product guides, review articles, technical papers, and brought out special issues of international journals. He is holding positions in the editorial board of many world renowned international journals. He has great experience in preparing B2B marketing, sales and advertising campaigns for leading IT Software Enterprises. $\mathrm{He}$ is expert in Cloud Computing, Business Intelligence and Analytics, Big Data, Market Research, Mobile Computing, ECommerce, ERP, Social Media Marketing/Advertising, E-Learning, Artificial Intelligence, Artificial Neural Networks, Machine Learning, Soft Computing, Telecommunications, Wireless Technology, Honeynet, and Networking Servers.

How to cite this paper: Sumit Goyal,"Artificial Neural Networks in Fruits: A Comprehensive Review", IJIGSP, vol.6, no.5, pp.53-63, 2014.DOI: 10.5815/ijigsp.2014.05.07 